CBPF-NF-041/97

hep-th/xxxxxxx

\title{
A No-Go Theorem for the Nonabelian Topological Mass Mechanism in Four Dimensions
}

\author{
M. Henneaux ${ }^{a, b}$, V.E.R. Lemes ${ }^{c}$, C.A.G. Sasaki ${ }^{c}$, S.P. Sorella ${ }^{d}$, \\ O.S. Ventura ${ }^{c}$ and L.C.Q. $\operatorname{Vilar}^{c}$ \\ ${ }^{a}$ Faculté des Sciences, Université Libre de Bruxelles, \\ Campus Plaine C.P. 231, B-1050 Bruxelles, Belgium \\ ${ }^{b}$ Centro de Estudios Científicos de Santiago, \\ Casilla 16443, Santiago 9, Chile \\ ${ }^{c}$ C.B.P.F, Centro Brasileiro de Pesquisas Físicas, \\ Rua Xavier Sigaud 150, 22290-180 Urca, Rio de Janeiro, Brazil \\ ${ }^{d}$ UERJ, Universidade do Estado do Rio de Janeiro, \\ Departamento de Física Teórica, Instituto de Física, UERJ, \\ Rua São Francisco Xavier, 524, 20550-013 Maracanã, Rio de Janeiro, Brazil
}

\begin{abstract}
We prove that there is no power-counting renormalizable nonabelian generalization of the abelian topological mass mechanism in four dimensions. The argument is based on the technique of consistent deformations of the master equation developed by G. Barnich and one of the authors. Recent attempts involving extra fields are also commented upon.
\end{abstract}




\section{Introduction}

One of the most intriguing issues of high energy physics is the understanding of the mechanism which provides the masses for the gauge vector bosons. As it is well known the Higgs mechanism, although consistent with the renormalizability and the unitarity constraints of quantum field theory, relies on the existence of scalar particles, the Higgs bosons, whose experimental evidence is still lacking.

Therefore any new alternative mechanism to generate masses is welcome, deserving attention and careful analysis. In particular, the idea that the vector boson masses could originate from a topological mechanism preserving exact gauge invariance is rather tempting and fascinating. The example provided by the topological nonabelian Chern-Simons term in three space-time dimensions is certainly the most celebrated way to provide a topological mass for the Yang-Mills fields [1]. In four dimensions, an analogous mechanism has been proposed in the abelian case [2]. It makes use of a two-form gauge field $B_{\mu \nu}=-B_{\nu \mu}$ suitably coupled to the one-form gauge connection $A_{\mu}$. The action reads

$$
S_{m}^{a b}=\int d^{4} x\left(-\frac{1}{4} F_{\mu \nu} F^{\mu \nu}-\frac{1}{12} H_{\mu \nu \rho} H^{\mu \nu \rho}+\frac{1}{2} m \varepsilon^{\mu \nu \rho \lambda} F_{\mu \nu} B_{\rho \lambda}\right),
$$

with $F_{\mu \nu}=\left(\partial_{\mu} A_{\nu}-\partial_{\nu} A_{\mu}\right)$ and $H_{\mu \nu \rho}$ the totally antisymmetric three-form

$$
H_{\mu \nu \rho}=\partial_{\mu} B_{\nu \rho}+\partial_{\nu} B_{\rho \mu}+\partial_{\rho} B_{\mu \nu}
$$

The action ([.]) is invariant under two kinds of local transformations given by

$$
\delta^{g} A_{\mu}=\partial_{\mu} \varepsilon, \quad \delta^{g} B_{\mu \nu}=0
$$

and

$$
\delta^{t} A_{\mu}=0, \quad \delta^{t} B_{\mu \nu}=\partial_{\mu} \varepsilon_{\nu}-\partial_{\nu} \varepsilon_{\mu} .
$$

Equations (I.3) and ([.4) correspond respectively to ordinary gauge transformations and to vector type transformations related to the tensorial character of the field $B_{\mu \nu}$. The parameter $m$ of the topological term $\varepsilon^{\mu \nu \rho \lambda} F_{\mu \nu} B_{\rho \lambda}$ in the expression (1.1) has the dimension of a mass. It is easily verified that this term gives a nonvanishing pole for the physical two-point function, yielding thus a gauge-invariant topological mass [2]. The two-form $B_{\mu \nu}$ is actually dual to the scalar field that is eaten up by the gauge field in the standard Higgs mechanism. There is, however, no additional particle and the action ([.1) describes a single massive vector field without Higgs boson.

The aim of this work is to discuss to what extent the abelian model (I.1) can be generalized to the nonabelian case. The analysis will be performed by making use of the method of the consistent deformations developed in [3]. We will end up, unfortunately, with a no-go theorem stating that it is not possible to generalize the 
expression (I.1) to a local, power counting renormalizable, nonabelian action while preserving the same field content and the same number of local symmetries. In other words, possible nonabelian generalizations of the action (I.1) will necessarely require non-renormalizable couplings, as in [四], or the introduction of extra fields [5].

The paper is organized as follows. In Sect.II we briefly review the method of consistent deformations. Sect.III will be devoted to the detailed proof of the aforementioned no-go theorem. Sect. IV discusses the assumptions of the no-go theorem and comments on possible ways out.

\section{Consistent Deformations}

The method that we shall follow is based on the antifield-BRST formalism and allows to construct interaction terms by consistently deforming the master equation!. Following the original work [3], the starting point is a given action $S_{i n v}\left[\phi^{a}\right]$ with local gauge symmetries

$$
\delta_{\varepsilon} \phi^{a}=R_{\alpha}^{a} \varepsilon^{\alpha}, \quad \delta_{\varepsilon} S_{i n v}\left[\phi^{a}\right]=0 .
$$

According to the well known antifield formalism (for a review appropriate to the subsequent cohomological considerations, see [8]), we introduce ghost fields $C^{\alpha}$ and a suitable set of antifields $\phi^{* A}$, so that the action

$$
S_{0}\left[\phi^{A}, \phi^{* A}\right]=S_{i n v}\left[\phi^{a}\right]+\int d^{4} x \phi_{a}^{*} R_{\alpha}^{a} C^{\alpha}+\ldots
$$

is a solution of the master equation

$$
\left(S_{0}, S_{0}\right)=\int d^{4} x \frac{\delta S_{0}}{\delta \phi^{A}} \frac{\delta S_{0}}{\delta \phi^{* A}}=0
$$

where $\phi^{A}=\left(\phi^{a}, C^{\alpha}\right)$ denote collectively all the fields and ghosts. The BRST differential $s$ in the space of the fields and antifields is defined through the antibraket

$$
s \phi^{A}=\left(\phi^{A}, S_{0}\right)=\frac{\delta S_{0}}{\delta \phi^{* A}}, \quad s \phi^{* A}=\left(\phi^{* A}, S_{0}\right)=\frac{\delta S_{0}}{\delta \phi^{A}} .
$$

Let us suppose now that the action $S_{0}$ refers to a free field theory which does not contain any coupling constant or mass parameter and let us ask ourselves if it is possible to introduce consistent interactions for $S_{0}$, i.e.

$$
S_{0} \rightarrow S=S_{0}+g_{i} S_{i}+g_{i} g_{j} S_{i j}+\ldots,
$$

\footnotetext{
${ }^{1}$ The usefulness of the deformation point of view (but not in the general framework of the antifield formalism, which allows off-shell open deformations of the algebra) has been advocated in [6]. For a recent discussion emphasizing the homological aspects, see [7].
} 
in such a way that the resulting action $S$ still satisfies the deformed master equation

$$
(S, S)=0
$$

Of course, due to locality and power counting, we shall limit ourselves to interactions $S_{i}$ which are integrated local polynomials in the fields and antifields with dimensions less or equal to four. Accordingly, the expansion parameters $g_{i}$ will be required to have nonnegative mass dimension. Therefore they will have the meaning of coupling constants and masses. As shown in ref. [3], the requirement of the validity of the master equation ([1.6) automatically implies the existence of a deformed action $S_{i n v}^{g}\left[\phi^{A}\right]$

$$
S_{i n v}^{g}\left[\phi^{A}\right]=S\left[\phi^{A}, \phi^{* A}=0\right]=S_{i n v}\left[\phi^{a}\right]+O\left(g_{i}\right),
$$

which is left invariant under a consistent deformed version of the original gauge symmetries, i.e.

$$
\delta_{\varepsilon}^{g} S_{i n v}^{g}\left[\phi^{A}\right]=0
$$

with

$$
\begin{aligned}
\delta_{\varepsilon}^{g} \phi^{a} & =\frac{\delta S}{\delta \phi^{* a}}\left[\phi^{A}, \phi^{* A}=0\right], \quad\left(C^{\alpha} \rightarrow \varepsilon^{\alpha}\right), \\
\delta_{\varepsilon}^{g} \phi^{a} & =R_{\alpha}^{a} \varepsilon^{\alpha}+O\left(g_{i}\right) .
\end{aligned}
$$

Equations ([I.7)-(II.9) mean indeed that we have been able to add invariant interaction terms to the original free action by suitable deforming the gauge symmetry, the construction being done order by order in the parameters $g_{i}$. For instance, in the case in which as the free action $S_{0}$ one chooses the Maxwell lagrangian, the above construction naturally leads to the nonabelian Yang-Mills action with the well known cubic and quartic interaction terms. Moreover, the usefulness of working with the master equation lies in the fact that the search of possible nontrivial interaction terms can be reduced to a cohomology problem for the free BRST differential of eq.(II.4), whose cohomology classes are eventually known or amenable to tractable computations. For a better understanding of this point, let us expand the master equation (II.6) in powers of the deformation parameters $g_{i}$ :

$$
\begin{aligned}
& \left(S_{0}, S_{0}\right)=0, \\
& \left(S_{0}, S_{i}\right)=0, \\
& 2\left(S_{0}, S_{i j}\right)+\left(S_{i}, S_{j}\right)=0,
\end{aligned}
$$

\footnotetext{
${ }^{2}$ According to ref [3], an interaction term is called trivial if it can obtained through a field redefinition.
} 
The first equation is nothing but the master equation for the free theory $S_{0}$, and is satisfied by hypothesis. From the second condition we see that $S_{i}$ has to be invariant under the action of the free BRST differential $s \equiv\left(., S_{0}\right)$. However, interactions of the type $S_{i}=\left(T_{i}, S_{0}\right)$, for some integrated local $T_{i}$, have to be neglected since they are seen to correspond to pure field redefinitions [3]. This means that the nontrivial interaction terms which can be added to the free action $S_{0}$ in the first order approximation in the parameters $g_{i}$ have to belong to the cohomology of the BRST differential $s$. Concerning the third equation, it is very easy to see that it can admit a solution only if the antibracket $\left(S_{i}, S_{j}\right)$ can be written in the form of an exact cocycle, i.e. $\left(S_{i}, S_{j}\right)=\left(T_{i j}, S_{0}\right)$, for some local $T_{i j}$. Otherwise, if $\left(S_{i}, S_{j}\right)$ belongs to the cohomology of $s$ we have an obstruction whose effect is to generate constraints among the various parameters $g_{i}$. The same conclusions hold for the higher order levels of the expansion of the deformed master equation ([1.6). In other words, at each step, the parameters $g_{i}$ are required to fulfill a certain number of conditions. It may happen that the conditions met at a certain level can be fulfilled only if some of the $g_{i}$ 's vanish. This means that the corresponding first-order deformation is obstructed, so that the full deformation program can be achieved only for a restricted susbset of the couplings. As we shall see in the next section, this will be the case of the nonabelian generalization we are looking for. In fact, we shall be able to prove that the abelian action (I.1) and the gauge symmetries of eqs. (I.3) and (I.4) can be consistently deformed only if $m g=0$ (where $g$ is the Yang-Mills coupling constant), implying that there is no nonabelian, power-counting renormalizable generalization of the topological mass term $\varepsilon^{\mu \nu \rho \lambda} F_{\mu \nu} B_{\rho \lambda}$.

\section{The No-Go Theorem}

Let us now apply the previous construction to the analysis of a possible nonabelian extension of the action (1.1). We shall start therefore with the following free gauge invariant action

$$
\Sigma_{0}=\int d^{4} x\left(-\frac{1}{4} F_{\mu \nu}^{a} F^{a \mu \nu}-\frac{1}{12} H_{\mu \nu \rho}^{a} H^{a \mu \nu \rho}\right),
$$

where $F_{\mu \nu}^{a}$ and $H^{a \mu \nu \rho}$ are the abelian curvatures ([.2) for a set of $n(a=1, . ., n)$ gauge and tensor fields $A_{\mu}^{a}$ and $B_{\mu \nu}^{a}$. It is worth recalling here that, within the consistent deformation set up, the mass parameter $m$ of eq.([.1) is considered, as any other coupling, as a deformation parameter. Accordingly, the topological mass term $\varepsilon^{\mu \nu \rho \lambda} F_{\mu \nu} B_{\rho \lambda}$ will in fact appear as a first order consistent deformation.

Of course, the free action ([II.1) is invariant under the gauge transformations

$$
\delta A_{\mu}^{a}=\partial_{\mu} \varepsilon^{a}, \quad \delta B_{\mu \nu}^{a}=\partial_{\mu} \varepsilon_{\nu}^{a}-\partial_{\nu} \varepsilon_{\mu}^{a} .
$$

Taking into account that the trasnformation of $B_{\mu \nu}^{a}$ is reducible, due to the existence of the zero modes $\delta \varepsilon_{\mu}^{a}=\partial_{\mu} \omega^{a}$, we introduce a set of ghosts $\left(c^{a}, \eta^{a \mu}, \rho^{a}\right)$, where $c^{a}$ 
and $\eta^{a \mu}$ stand for the ghost corresponding to the gauge transformations ([II.2) and $\rho^{a}$ is a ghost for ghost accounting for the reducibility. Moreover, introducing also the antifields $\left(A_{a}^{* \mu}, B_{a}^{* \mu \nu}, c_{a}^{*}, \eta_{a}^{* \mu}, \rho_{a}^{*}\right)$ and the corresponding action $\Sigma_{a n t}$

$$
\Sigma_{\text {ant }}=\int d^{4} x\left(A_{a}^{* \mu} \partial_{\mu} c^{a}+B_{a}^{* \mu \nu} \partial_{\mu} \eta_{\nu}^{a}+\eta_{a}^{* \mu} \partial_{\mu} \rho^{a}\right)
$$

one finds that the complete free action

$$
S_{0}=\Sigma_{0}+\Sigma_{\text {ant }}
$$

satisfies the master equation

$$
\left(S_{0}, S_{0}\right)=0
$$

with

$$
\left(S_{0}, S_{0}\right)=\int d^{4} x\left(\frac{\delta S_{0}}{\delta A^{a \mu}} \frac{\delta S_{0}}{\delta A_{a \mu}^{*}}+\frac{1}{2} \frac{\delta S_{0}}{\delta B^{a \mu \nu}} \frac{\delta S_{0}}{\delta B_{a \mu \nu}^{*}}+\frac{\delta S_{0}}{\delta c^{a}} \frac{\delta S_{0}}{\delta c_{a}^{*}}+\frac{\delta S_{0}}{\delta \eta^{a \mu}} \frac{\delta S_{0}}{\delta \eta_{a \mu}^{*}}+\frac{\delta S_{0}}{\delta \rho^{a}} \frac{\delta S_{0}}{\delta \rho_{a}^{*}}\right) .
$$

According to Eq.(III.4), the nilpotent BRST transformation is

$$
\begin{array}{ll}
s A_{\mu}^{a}=\partial_{\mu} c^{a}, & s A_{a}^{* \mu}=\partial_{\nu} F^{a \nu \mu}, \\
s B_{\mu \nu}^{a}=\partial_{\mu} \eta_{\nu}^{a}-\partial_{\nu} \eta_{\mu}^{a}, & s B_{a}^{* \mu \nu}=\partial_{\rho} H_{a}^{\rho \mu \nu}, \\
s \eta_{\mu}^{a}=\partial_{\mu} \rho^{a}, & s \eta_{a}^{* \mu}=-\partial_{\nu} B_{a}^{* \mu \nu}, \\
s c^{a}=0, & s c_{a}^{*}=-\partial_{\mu} A_{a}^{* \mu}, \\
s \rho^{a}=0, & s \rho_{a}^{*}=\partial_{\mu} \eta_{a}^{* \mu} .
\end{array}
$$

We should remark that the antifields $\left(c_{a}^{*}, \rho_{a}^{*}\right)$ conjugate to the ghosts $\left(c^{a}, \rho^{a}\right)$, although not explicitely appearing in the expression (III.3) due to the fact that $c^{a}$ and $\rho^{a}$ do not transform under $s$ in the free abelian limit, are needed to allow a priori for general deformations deforming also the gauge algebra or the reducibility functions [3]. Let us display, for further use, the quantum numbers of all the fields and antifields.

\begin{tabular}{|c|c|c|c|c|c|c|c|c|c|c|}
\hline & $A_{\mu}^{a}$ & $B_{\mu \nu}^{a}$ & $c^{a}$ & $\eta_{\mu}^{a}$ & $\rho^{a}$ & $A_{\mu}^{* a}$ & $B_{\mu \nu}^{* a}$ & $c^{* a}$ & $\eta_{\mu}^{* a}$ & $\rho^{* a}$ \\
\hline$N_{g}$ & 0 & 0 & 1 & 1 & 2 & -1 & -1 & -2 & -2 & -3 \\
\hline $\operatorname{dim}$ & 1 & 1 & 0 & 0 & -1 & 3 & 3 & 4 & 4 & 5 \\
\hline
\end{tabular}

Table 1: Ghost numbers and dimensions. 
We face now the problem of characterizing the possible interactions terms $S_{i}$ that can be added to the action ([III.4) while preserving the master equation (III.5). Following the algebraic set up of the previous Section, the consistent interactions which can be introduced to the first order in the deformation parameters are nontrivial solutions of the consistency condition

$$
\left(S_{0}, S_{i}\right)=0
$$

with $S_{i}$ local integrated polynomials of ghost number zero and dimension bounded by four.

The solutions of ([II.8) for free $p$-form gauge fields have been studied in [9] and fall into three categories: (i) those that do not deform the gauge symmetry; (ii) those that deform the gauge transformations but not the gauge algebra; and (iii) those that deform both the gauge transformations and the gauge algebra.

The first category contains the gauge-invariant functions (= functions of the curvature components and their derivatives) as well as the Chern-Simons terms. The only candidates allowed by Lorentz invariance and power-counting are the kinetic terms $\sim F^{2}, H^{2}$ - which are already present in the Lagrangian - and the Chern-Simons term $\varepsilon^{\mu \nu \sigma \lambda} B_{\mu \nu}^{a} F_{\sigma \lambda}^{a}$ (the Chern-Simons term $\varepsilon^{\mu \nu \sigma \lambda} H_{\mu \nu \sigma}^{a} A_{\lambda}^{a}$ differs from $\varepsilon^{\mu \nu \sigma \lambda} B_{\mu \nu}^{a} F_{\sigma \lambda}^{a}$ by a total derivative and hence is not independent from it). Consequently, there is only one independent new vertex in the first category, which we denote by $S_{2}$,

$$
S_{2}=m_{a b} \int d^{4} x\left(\varepsilon^{\mu \nu \sigma \lambda} B_{\mu \nu}^{a} F_{\sigma \lambda}^{b}\right) .
$$

Here, $m_{a b}$ is a mass matrix which may have zero eigenvalues, reflecting the possibility that some gauge bosons may remain massless.

The second category involves interactions of the Noether form $A_{\mu}^{a} j_{a}^{\mu}$ and $B_{\mu \nu}^{a} k_{a}^{\mu \nu}$, where $j_{a}^{\mu}$ are gauge-invariant conserved currents, while $k_{a}^{\mu \nu}$ are gauge-invariant conserved antisymmetric tensors $\left(\partial_{\mu} k_{a}^{\mu \nu}=0\right)$. There are only two types of non-trivial gauge-invariant conserved antisymmetric tensors, which are $F_{a}^{\mu \nu}$ and $\epsilon^{\mu \nu \rho \sigma} \bar{H}_{\rho}^{a} \bar{H}_{\sigma}^{b}[9]$. Here, $\bar{H}^{a}$ denotes the one-form dual to $H^{a}$. The conserved tensors $\epsilon^{\mu \nu \rho \sigma} \bar{H}_{\rho}^{a} \bar{H}_{\sigma}^{b}$, which lead to the Freedman-Townsend coupling [1, are excluded by power-counting renormalizability since they require coupling constants with dimensions of an inverse mass. This leaves us with $B_{\mu \nu}^{a} F^{b \mu \nu}$ only. Similary, even though there is an infinite number of gauge invariant conserved currents $j_{\mu}^{a}$, Lorentz invariance and power-counting renormalizability exclude all couplings of the form $A_{\mu}^{a} j_{a}^{\mu}$ except $t_{a b} A_{\mu}^{a} \varepsilon^{\mu \nu \sigma \lambda} H_{\mu \nu \sigma}^{b}$ and $t_{a b} A_{\mu}^{a} \partial_{\nu} F^{b \mu \nu}$. But $t_{a b} A_{\mu}^{a} \varepsilon^{\mu \nu \sigma \lambda} H_{\mu \nu \sigma}^{b}$ is equivalent to the above Chern-Simons term, while $t_{a b} A_{\mu}^{a} \partial_{\nu} F^{b \mu \nu}$ vanishes on-shell and can be absorbed by redefinitions. To summarize, there is accordingly only one novel coupling in the second category, namely $\mu_{a b} B_{\mu \nu}^{a} F^{b \mu \nu}$, with matrix $\mu$ having the dimensions of a mass. This coupling is of the Chapline-Manton type [10] and has the following BRST invariant 
extension,

$$
S_{3}=\mu_{a b} \int d^{4} x\left(\frac{1}{2} B_{\mu \nu}^{a} F^{b \mu \nu}+A^{* a \mu} \eta_{\mu}^{b}+c^{* a} \rho^{b}\right) .
$$

It is rather interesting to remark that the expression (III.10) allows, at least in the abelian case, for a further nontopological mass mechanism. It is indeed immediate to check that the following abelian action

$$
\begin{aligned}
S_{\mu}^{a b} & =\int d^{4} x\left(-\frac{1}{4}\left(F_{\mu \nu}-\mu B_{\mu \nu}\right)^{2}+\frac{1}{12} H_{\mu \nu \rho} H^{\mu \nu \rho}\right) \\
& =\int d^{4} x\left(-\frac{1}{4} F_{\mu \nu} F^{\mu \nu}+\frac{1}{12} H_{\mu \nu \rho} H^{\mu \nu \rho}+\frac{\mu}{2} B^{\mu \nu} F_{\mu \nu}-\frac{\mu^{2}}{4} B_{\mu \nu} B^{\mu \nu}\right),
\end{aligned}
$$

is left invariant by the transformations

$$
\delta A_{\mu}=\partial_{\mu} \varepsilon+\mu \varepsilon_{\mu}, \quad \delta B_{\mu \nu}=\partial_{\mu} \varepsilon_{\nu}-\partial_{\nu} \varepsilon_{\mu} .
$$

The new terms in (III.11) provide a nontopological mass for the two-form field $B_{\mu \nu}$. The action (III.11) was actually already considered in [11]. Note that the transformation of the gauge connection gets modified by an extra $\mu$-dependent term which enables one to gauge it away. Recall also that a massive two-form in four dimensions describes massive spin-one particles, just as a massive one-form.

Finally, the third category contains only the familiar Yang-Mills cubic interaction vertex with dimensionless parameter $g$ [9] (second reference),

$$
S_{1}=g \int d^{4} x f^{a b c}\left(-\frac{1}{2} F_{\mu \nu}^{a} A^{b \mu} A^{c \nu}+A_{\mu}^{* a} A^{b \mu} c^{c}+\frac{1}{2} c^{* a} c^{b} c^{c}\right) .
$$

It should be stressed, in particular, that (III.13) cannot be accompanied by a deformation of the type $A_{\mu}^{a} B_{\rho \sigma}^{b} H^{c \mu \rho \sigma} f_{a b c}$. Such an interaction vertex is the minimal coupling to the Yang-Mills field of a charged $B$-field transforming in the adjoint. It has the Noether form $A_{\mu}^{a} j_{a}^{\mu}$ where the current $j_{a}^{\mu}=B_{\rho \sigma}^{b} H^{c \mu \rho \sigma} f_{a b c}$ is, however, not gauge invariant under the gauge transformations of the two-forms. For this reason, $A_{\mu}^{a} B_{\rho \sigma}^{b} H^{c \mu \rho \sigma} f_{a b c}$ is also not gauge-invariant, even up to a total derivative, and does not define an observable. Thus, it does not lead to a consistent deformation. The two-forms $B_{\rho \sigma}^{a}$ are uncharged [12] and can only couple to the connection $A$ through Chern-Simons or Chapline-Manton terms (to first order).

Having characterized the possible nontrivial consistent interaction terms that can be added to first order in the deformation parameters $(g, m, \mu)$, let us turn to the study of the higher order consistency conditions stemming from the requirement of validity of the deformed master equation to all orders. As we have already seen, the second order consistency condition, 1.e. the third equation of the system ([I.10), can be solved only if the antibrackets $\left(S_{i}, S_{j}\right)$, with $i, j=1,2,3$, can be written as exact BRST cocycles. The antibracket $\left(S_{2}, S_{2}\right)$ is automatically vanishing, due to the fact that $S_{2}$ is independent from the antifields. In addition, the constraints which follow 
from $\left(S_{1}, S_{1}\right)$ are the usual ones which lead to the pure Yang-Mills vertices and are satisfied by identifying the $f^{a b c}$ 's in eq.([II.13) with the structure constant of a Lie group. Concerning now the antibrackets $\left(S_{2}, S_{3}\right)$ and $\left(S_{3}, S_{3}\right)$, they are easily seen to be BRST trivial

$$
\begin{aligned}
& \left(S_{2}, S_{3}\right)=-\frac{2}{3} m \mu \int d^{4} x \varepsilon^{\mu \nu \rho \sigma} H_{\mu \nu \rho}^{a} \eta_{\sigma}^{a}=s\left(\frac{m \mu}{2} \int d^{4} x \varepsilon^{\mu \nu \rho \sigma} B_{\mu \nu}^{a} B_{\rho \sigma}^{a}\right) \\
& \left(S_{3}, S_{3}\right)=-2 \mu^{2} \int d^{4} x \partial^{\mu} B_{\mu \nu}^{a} \eta^{a \nu}=s\left(\frac{\mu^{2}}{2} \int d^{4} x B^{a \mu \nu} B_{\mu \nu}^{a}\right)
\end{aligned}
$$

so that they do not bring any obstruction. It remains therefore to analyse the terms $\left(S_{1}, S_{3}\right)$ and $\left(S_{1}, S_{2}\right)$, given respectively by

$$
\begin{aligned}
& \left(S_{1}, S_{3}\right)=-g \mu \int d^{4} x f^{a b c}\left(F_{\mu \nu}^{a} A^{b \mu} \eta^{c \nu}-\partial^{\mu}\left(A_{\mu}^{b} A_{\nu}^{c}\right) \eta^{a \nu}+\left(\partial^{\mu} B_{\mu \nu}^{a}\right) A^{b \nu} c^{c}\right. \\
& \left.-A_{\mu}^{* a} c^{c} \eta^{b \mu}-A_{\mu}^{* a} A^{b \mu} \rho^{c}-c^{* a} c^{b} \rho^{c}\right) \\
& \left(S_{1}, S_{2}\right)=-\frac{2}{3} m g \int d^{4} x f^{a b c} \varepsilon^{\mu \nu \rho \sigma} H_{\mu \nu \rho}^{a} A_{\sigma}^{b} c^{c} .
\end{aligned}
$$

It is not difficult to convince oneself that the above expressions are not BRST trivial, representing thus a real obstruction to the deformation of the master equation. Concerning the first expression, we indeed observe that the antifield dependent terms do not contain any space-time derivative. On the other hand, from eqs. (III.7) we see that the BRST transformations of the antifields always introduce a spacetime derivative, implying thus that $\left(S_{1}, S_{3}\right)$ cannot be cast in the form of a pure $s$-variation. Similarly, by means of a simple counting of the space-time derivatives appearing in the second term of eq.(III.15) as well as in the BRST transformations (III.7), we can easily verify that the only possible candidate whose BRST variation could reproduce the second antibracket $\left(S_{1}, S_{2}\right)$ is given by

$$
\int d^{4} x f^{a b c} \varepsilon^{\mu \nu \rho \sigma} B_{\mu \nu}^{a} A_{\rho}^{b} A_{\sigma}^{c}
$$

However, from

$$
s \int d^{4} x f^{a b c} \varepsilon^{\mu \nu \rho \sigma} B_{\mu \nu}^{a} A_{\rho}^{b} A_{\sigma}^{c}=2 \int d^{4} x f^{a b c} \varepsilon^{\mu \nu \rho \sigma}\left(\partial_{\mu} \eta_{\nu}^{a} A_{\rho}^{b} A_{\sigma}^{c}+B_{\mu \nu}^{a} \partial_{\rho} c^{b} A_{\sigma}^{c}\right) .
$$

we see that the variation of (III.16) unavoidably contains $\eta_{\nu}^{a}$, so that $\left(S_{1}, S_{2}\right)$ is a nontrivial element of the BRST cohomology.

It follows then that the only way to consistently implement the master equation to second order in the deformation parameters is to let the coefficients of the 
nontrivial antibrackets ([II.15) vanish, 1.e.

$$
\begin{aligned}
& g \mu=0, \\
& g m=0 .
\end{aligned}
$$

This means that, as long as we insist in adding the Yang-Mills coupling to the free action, then necessarily $\mu=0$ and $m=0$. Otherwise, we can keep the massive terms $\mu$ and $m$ but the Yang-Mills interaction is irremediably lost, and we are left with the two massive abelian models of eqs.([.1) and (III.11) for a set of $n$ noninteracting fields. [Both mass terms can in fact be consistently considered simultaneously because both $\left(S_{2}, S_{3}\right)$ and $\left(S_{3}, S_{3}\right)$ are BRST-exact].

Therefore, it is not possible to generalize the massive action (1.1) to a nonabelian local, power counting renormalizable, interacting theory while preserving the same field content and the same set of local symmetries. This concludes the proof of the no-go theorem on the introduction of a topological mass in four dimensions for a non-abelian group. This obstruction is in sharp contrast with the three-dimensional Chern-Simons construction that allows any gauge group with an invariant metric;.

\section{Comments}

As any no-go theorem, our result is no stronger than the assumptions underlying it. These are two-fold. First, we have excluded non power-counting renormalizable couplings since the goal is to construct an alternative to the Higgs mechanism with the same good quantum properties. If one relaxes this condition - and this is suggested by the more modern approach to renormalization advocated in [14] -, one can construct a Lagrangian that incorporates both the topological mass term and the Yang-Mills coupling. This Lagrangian contains also the non power-counting renormalizable Freedman-Townsend interaction and has been written down explicitly in [4] (see also [15]). It contains two independent coupling constants, one, $m$, with the dimensions of a mass and the other, $\alpha$, with the dimensions of an inverse mass. It reads

$$
L=\frac{1}{4} \varepsilon^{\mu \nu \rho \sigma} B_{\mu \nu}^{a} \Phi_{\rho \sigma}^{a}-\frac{1}{2} \beta^{a \mu} \beta_{\mu}^{a}-\frac{1}{2} F_{\mu \nu}^{a} F_{\mu \nu}^{a}
$$

where $\beta^{a \mu}$ is an auxiliary field equal on-shell to the dual of $H^{a}$ in the free limit. In (IV.1]), $F_{\mu \nu}^{a}$ is the standard non-abelian field strength

$$
F_{\mu \nu}^{a}=\partial_{\mu} A_{\nu}^{a}-\partial_{\nu} A_{\mu}^{a}+g f_{b c}^{a} A_{\mu}^{b} A_{\nu}^{c}, \quad g=m \alpha,
$$

\footnotetext{
${ }^{3}$ Our analysis provides another instance where a duality transformation (here between the twoform $B$ and the scalar field of the Higgs mechanism) that can be defined in the abelian case cannot be implemented in the non-abelian extension 13 .
} 
while $\Phi_{\mu \nu}^{a}$ is given by a similar expression with $\beta_{\mu}^{a}+m A_{\mu}^{a}$ in place of $A_{\mu}^{a}$ and $\alpha$ in place of $g$,

$$
\Phi_{\mu \nu}^{a}=\partial_{\mu} \beta_{\nu}^{a}-\partial_{\nu} \beta_{\mu}^{a}+m\left(\partial_{\mu} A_{\nu}^{a}-\partial_{\nu} A_{\mu}^{a}\right)+\alpha f_{b c}^{a}\left(\beta_{\mu}^{b}+m A_{\mu}^{b}\right)\left(\beta_{\nu}^{c}+m A_{\nu}^{c}\right) .
$$

The first order deformations are here the topological mass term $m \varepsilon^{\mu \nu \rho \sigma} B_{\mu \nu}^{a}\left(\partial_{\rho} A_{\sigma}^{a}-\right.$ $\left.\partial_{\sigma} A_{\rho}^{a}\right)$ and the Freedman-Townsend vertex $\alpha f_{a b c} \varepsilon^{\mu \nu \rho \sigma} B_{\mu \nu}^{a} \beta_{\rho}^{b} \beta_{\sigma}^{c}$. The Yang-Mills coupling emerges at second order, the Yang-Mills coupling constant $g$ being related to the topological mass $m$ and the Freedman-Townsend coupling constant $\alpha$ through $g=m \alpha$. The Lagrangian (IV.1) contains terms of up to fourth order in the coupling constants. Of course, one could equivalently include the mass term in the starting free lagrangian. In that case, the expansion in the coupling constant stops at second order (see [16] for a first order formulation along these lines).

The second assumption underlying our analysis is that the interacting theory possesses the same field content and the same number of independent gauge symmetries as the free theory. This requirement appears to be necessary in order for perturbation theory about the quadratic piece of the action to be carried out. Now, one may also give up this second assumption. This has been done in [5], where a non-abelian generalization of the abelian action ([.1) has been proposed, in which the abelian field strengths $F$ are replaced by the non-abelian ones, and the derivatives of $B$ are replaced by covariant derivatives (the two-form $B$ transforming in the adjoint). This generalization is invariant under the standard Yang-Mills gauge transformations, but not under (I.4) or any generalization thereof since it corresponds to introducing a minimal coupling of the two-form $B$ to the Yang-Mills connection $A$, and this was excluded above. The generalization does not provide a "consistent deformation" of the abelian theory because it has less gauge symmetries. This does not mean, however, that it is physically inconsistent, but that the free theory cannot be used straightforwardly as a starting point for the standard perturbative expansion (obscuring in particular the meaning of power-counting perturbative renormalizability). Actually, the Hamiltonian analysis performed along the conventional Dirac lines - or any other equivalent method - is rather direct and seems to indicate that the theory is acceptable, although there are difficulties related to the fact that some Poisson bracket matrices have varying ranks in phase space.

One may reinstate (a non-abelian version of) the gauge symmetry ([.4) by adding an extra field transforming appropriately [5]. But even after this is done, the theory does not have the same number of gauge symmetries as the abelian limit, since in that limit, the extra field disappears from the Lagrangian: the abelian theory with the additional field has the additional gauge freedom of shifting independently the extra field. Our no-go theorem on the absence of deformations preserving all the gauge symmetries of the free theory applies in fact to any equivalent formulation of the abelian starting point that can be obtained by adding extra auxiliary or pure gauge fields, as the cohomological theorems of [17] (section 15) indicate.

A construction similar to the above mass generation mechanism has been pro- 
posed recently in three dimensions by Jackiw and Pi [18]. What plays there the role of the two-form $B_{\mu \nu}^{a}$ is a vector field $B_{\mu}^{a}$. An analog of the above no-go theorem for the non-abelian case has been demonstrated long ago in [19]. The authors of [18 analyse the non-abelian theory with less gauge symmetries that one obtains by minimally coupling the additional vector field $B_{\mu}^{a}$ to $A_{\mu}^{a}$. The resulting theory suffers from the same difficulties as the theory of [5] since a standard perturbation expansion about the free limit cannot be carried out. Whether this theory, or the four-dimensional version of [5], can be quantized in a tractable and meaningful way is therefore still an open question. This point deserves further investigation in view of the attractive features of the model exhibited in [18.

\section{Acknowledgments}

$\mathrm{MH}$ is grateful to Bernard Julia for pointing out to him reference 15. He also thanks the Physics Departments of UERJ, UFRJ and CBPF (Rio) for kind hospitality while this work was being carried out. Finally, the Conselho Nacional de Pesquisa e Desenvolvimento, $\mathrm{CNP} q$ Brazil, is gratefully acknowledged for financial support.

\section{References}

[1] R. Jackiw and S. Templeton, Phys. Rev. D23 (1981) 2291; W. Siegel, Nucl. Phys. B156 (1979) 135; J.F. Schonfeld, Nucl. Phys. B185 (1981) 157; S. Deser, R. Jackiw and S. Templeton, Ann. Phys. (N.Y.) 140 (1982) 372;

[2] E. Cremmer and J. Scherk, Nucl. Phys. B72(1974) 117; C.R. Hagen, Phys. Rev. D19 (1979) 2367; T.J. Allen, M.J. Bowick and A. Lahiri, Mod. Phys. Lett. A6 (1991)559; R. Amorim and J. Barcelos-Neto, Mod. Phys. Lett. A10 (1995) 917;

[3] G. Barnich and M. Henneaux, Phys. Lett. B311 (1993) 123;

[4] D. Z. Freedman and P. K. Townsend, Nucl. Phys. B177 (1981) 282;

[5] A. Lahiri, Generating Vector Boson Masses, hep-th/9301060; A. Lahiri, Phys. Rev. 55 (1997) 5045; D.S. Hwang and C. Y. Lee, J. Math. Phys. 38 (1997) 30; J. Barcelos-Neto, A. Cabo and M.B.D. Silva, Z. Phys. C72 (1996) 345; J. BarcelosNeto and S. Rabello, Mass generation for gauge fields in the Salam-Weinberg theory without Higgs bosons, to appear in Z. Phys. C, hep-th/9601076; 
[6] B. Julia, in Recent Developments in Quantum Field Theory, J. Ambjorn, B.J. Durhuus and J. L. Petersen eds, Elsevier (1985) pp 215-225; B. Julia, in Topological and Geometrical Methods in Field Theory, J. Hietarinta and J. Westerholm eds, World Scientific (1986) pp325-339;

[7] J. Stasheff, Deformation Theory and the Batalin-Vilkovisky Master Equation, q-alg/9702012;

[8] M. Henneaux and C. Teitelboim, Quantization of Gauge Systems, Princeton University Press, Princeton, NJ, 1992;

[9] M. Henneaux, Phys. Lett. 368B (1996) 83; M. Henneaux, B. Knaepen and C. Schomblond, Comm. Math. Phys. 186 (1997) 137; M. Henneaux and B. Knaepen, All consistent interactions for exterior form gauge fields, hepth/9706119;

[10] G.F. Chapline and N.S. Manton, Phys. Lett. B120 (1983) 105; H. Nicolai and P.K. Townsend, Phys. Lett. 98B (1981) 257; A. H. Chamseddine, Nucl. Phys. B185 (1981) 403; Phys. Rev. D24 (1981) 3065; E. Bergshoeff, M. de Roo, B. de Wit and P. van Nieuwenhuizen, Nucl. Phys. B195 (1982) 97;

[11] M. Kalb and P. Ramond, Phys. Rev. D9 (1974) 2273;

[12] S. Deser and E. Witten, Nucl. Phys. 178 (1981) 491;

[13] S. Deser and C. Teitelboim, Phys. Rev. D 13 (1976) 1572;

[14] J. Gomis and S. Weinberg, Nucl. Phys. B469 (1996) 473; S. Weinberg, The Quantum Theory of Fields, volumes I and II, Cambridge University Press, Cambridge 1995 and 1996;

[15] J. Thierry-Mieg, Nucl. Phys. B335 (1990) 334;

[16] A. Khoudeir, Mod. Phys. Lett. A11 (1996) 2489;

[17] G. Barnich, F. Brandt and M. Henneaux, Commun.Math.Phys. 174 (1995) 57;

[18] R. Jackiw and S.-Y. Pi, Phys. Lett. 403B (1997) 297;

[19] R. Arnowitt and S. Deser, Nucl. Phys. 49 (1963) 133. 